\title{
Concurrent infections with multiple human papillomavirus (HPV) types in the New Technologies for Cervical Cancer (NTCC) screening study
}

\author{
Francesca Carozzi ${ }^{a}$, Guglielmo Ronco ${ }^{b, *}$, Anna Gillio-Tos c, Laura De Marco c, \\ Annarosa Del Mistro ${ }^{d}$, Salvatore Girlando ${ }^{e}$, Silvia Franceschi ${ }^{f}$, \\ Martyn Plummer ${ }^{f}$, Salvatore Vaccarella ${ }^{f}$, For the New Technologies \\ for Cervical Cancer screening (NTCC) Working Group ${ }^{g}$ \\ a Centre for Cancer Study and Prevention, Via Cosimo il Vecchio 2, 50139 Florence, Italy \\ b Centre for Cancer Prevention (CPO), Via San Francesco da Paola 31, 10128 Turin, Italy \\ c Cancer Epidemiology Unit - CERMS, University of Turin, Turin, Italy \\ d Venetian Oncology Institute, IRCCS, Via Gattamelata 64, 35128 Padova, Italy \\ e S. Chiara Hospital, Largo Medaglie d'Oro, 38123 Trento, Italy \\ ${ }^{\mathrm{f}}$ International Agency for Research on Cancer, 150 cours Albert Thomas, 69372 Lyon Cedex 08, France
}

\section{A R T I C L E I N F O}

Article history:

Available online 14 November 2011

Keywords:

Human papillomavirus

Prevalence

Women

Multiple infections

GP5+/6+ PCR

\begin{abstract}
A B S T R A C T
Introduction: We investigated clustering patterns of human papillomavirus (HPV) in a large study, the New Technologies in Cervical Cancer (NTCC) screening study.

Materials and Methods: Women aged 25-60 years who attended cervical screening in eight different areas in Northern and Central Italy were tested for HPV infection with Hybrid Capture 2 (HC2). Genotyping of the HC2-positive samples was performed for 13 HPV types targeted by GP5+/GP6+ PCR, followed by Reverse Line Blot. Logistic regression was used to model type-specific HPV positivity, adjusted for age, study area, and specific HPV type prevalence. Subject-level random effects were added to represent unobservable risk factors common to all HPV types. Results: A total of 36,877 women were included. Of 2833 HC2-positive women, 2108 were confirmed to be positive for any of the 13 specific HPV infections using the PCR assay, and amongst them 430 (20.4\% of all PCR-positive women) were infected with multiple types. The observedto-expected ratio for infection with $\geqslant 2$ HPV types was 1.21 (95\% Credible Interval: 1.13-1.30). Amongst the 78 combinations of specific HPV types, none of the pairs reached the chosen level of significance, $p$-value $<0.01$.

Conclusions: Multiple HPV infections occurred more frequently than predicted by chance. The excess of multiple infections was small, though not completely absent, after controlling for all sources of common correlation between HPV types. The present analysis of the NTCC screening study showed no evidence that specific HPV types have the tendency to be found more or less often than others in coinfections.
\end{abstract}

(c) 2011 Elsevier Ltd. All rights reserved.

\footnotetext{
* Corresponding author: Tel.: +39 0116333050; fax: +39 0116333861.

E-mail address: guglielmo.ronco@cpo.it (G. Ronco).

${ }^{g}$ For the New Technologies for Cervical Cancer screening (NTCC) Working Group. The following are contributing members of the NTCC Working Group: Turin: N. Segnan and R. Rizzolo (CPO Piemonte, Turin); Trento: P. Dalla Palma, E. Polla, and A.M. Piccolo (Unit of Pathology, S. Chiara Hospital); Veneto: M. Zorzi, H. Frayle-Salamanca, and L. Baboci, (Veneto Oncology Institute IRCCS); Emilia-Romagna: C. Naldoni (Emilia Romagna Region); S. Folicaldi and M. De Lillo (Unit of Pathology, Presidio Ospedaliero, AUSL di Imola); P. Schincaglia (Centre for Cancer Prevention, Ravenna); Florence: M. Confortini, M. Zappa and E. Burroni (ISPO, Florence); Lazio: P. Giorgi-Rossi (ASP Lazio, Rome). $0959-8049 / \$$ - see front matter @ 2011 Elsevier Ltd. All rights reserved.
}

doi:10.1016/j.ejca.2011.10.010 


\section{Introduction}

Concomitant infections with multiple human papillomavirus (HPV) genotypes are common in cervicovaginal cell samples, especially in young women and women with multiple sexual partners. ${ }^{1-8}$ To understand whether certain HPV types have the tendency to be involved in multiple infections beyond or below what would be expected by sexual transmission and common risk factors is of relevance for the evaluation of the effects of HPV prophylactic vaccines. The removal of certain HPV types through vaccination could, in theory, indirectly increase or decrease the prevalence of other untargeted types.

Previous studies found a general tendency of all HPV types to cluster together, which was explained with the fact that the routes of transmission and risk factors are the same for all HPV types. However, few studies only have analysed two-way associations between HPV types, and their interpretation has often been hampered by limitations in sample size. ${ }^{1-7,9-12}$

We, therefore, investigated the patterns of HPV coinfections in a large study, the New Technologies in Cervical Cancer (NTCC) screening study, that used GP5+/6+ PCR as HPV detection method and Reverse Line Blot (RLB) as genotyping procedure.

\section{Methods}

The NTCC study was conducted within the framework of organised cervical cancer screening programmes in Northern and Central Italy (Turin, Trento, Padua, Florence, Bologna, Imola, Ravenna and Viterbo). Methods of recruitment and randomisation have been described previously. ${ }^{13-15}$ Briefly, following an invitation sent to the entire screening target population, women aged 25-60 years attending a new round of cervical screening were randomly assigned to conventional cytology or to an experimental arm that followed two phases depending on the period of recruitment. In the first phase (February 2002-June 2003), women assigned to the experimental arm were tested for HPV infection plus liquid-based cytology, while in the second phase (July 2003-December 2004) they were tested for HPV only. Women were excluded if they were pregnant, reported to be virgin, had undergone hysterectomy, or had been treated for cervical intraepithelial neoplasia (CIN) or cancer in the past 5 years. $74.0 \%$ of eligible women accepted to be randomised. Overall, 47,369 women that were assigned to the experimental arm were considered for the present analysis. Women were referred to colposcopy and biopsy according to an algorithm previously described. ${ }^{13,15}$ All participants provided written informed consent, and the study was approved by the local ethics committees of participating centres.

\subsection{HPV testing and genotyping}

Cervical specimens were collected using a plastic Ayre's spatula and a cytobrush, and eluted into the PreservCyt buffer (phase 1) or in the standard transport medium (Qiagen Incorporated) (phase 2). The presence of HPV in cervical specimens was evaluated by Hybrid Capture 2 (HC2; Digene Corporation, Gaithersburg, MD, USA) using probes designed to detect oncogenic HPV types 16, 18, 31, 33, 35, 39, 45, 51, 52, 56, 58, 59, and 68. For 37,367 women of the experimental arm that were randomised in both study phases in Turin, Trento, Padua and Florence and in study phase 2 only in Bologna, Imola, Ravenna and Viterbo, genotyping of the HC2-positive samples was put in place.

Amongst them, a total of 37,077 women had a valid HC2 result and 3,033 were found to be HPV-positive. One hundred and thirteen HPV-positive women were excluded from the present analysis because the corresponding samples for genotyping were not available, leaving 2920 samples available for genotyping. Genotyping was performed by GP5+/GP6+ PCR, followed by RLB. ${ }^{16,17}$ Although GP5+/6+ PCR can in principle detect $>40$ HPV types, in this analysis HPV genotyping was restricted to the $13 \mathrm{HPV}$ types included in the HC2.

\subsection{Statistical analysis}

A multivariate logistic regression model was used to model type-specific HPV positivity, as previously described. ${ }^{1}$ The presence of each HPV type was considered as a separate outcome for each woman. Covariates in the model included age, study area and specific HPV type prevalence. As the data have a hierarchical structure, with HPV infections nested within women, multilevel models were used, with woman-level random effects. In this context, a random effect is an unobserved quantity that varies between women. Random effects account for the fact that women with the same observable risk factors may have different levels of risk for prevalent HPV infection and that type-specific HPV measurements in the same woman are correlated with each other. Woman-level random effects represent residual sources of common correlation between all HPV types other than those already represented by the covariates, that is, unobserved host or sexual behavioural risk factors. Woman-level random effects are particularly useful to overcome the limitations of questionnaire variables (that is, lack of availability and relatively poor quality of certain variables, notably on sexual behaviour) as predictors of HPV positivity. Models were fitted using a Bayesian approach with Markov Chain Monte Carlo (MCMC) simulation. Estimates were reported as posterior means and 95\% credible intervals (95\% Credible Interval (CI)). Discrepancies between the data and the model were assessed by posterior predictive two-sided $p$-values and measured by observed-to-expected (O/E) ratios for joint HPV infections. ${ }^{18}$ Because all possible two-way interactions between the 13 HPV types were tested, this generated 78 -values. When testing 78 different hypotheses, one would expect approximately 4 falsely positive associations due to chance with a significance level of 0.05 , and $<1$ with a significance level of 0.01 . Therefore, to minimise errors due to multiple comparisons and for consistency with previous analyses, ${ }^{1}$ the threshold for significance of $p$-values to test type-type associations was set to 0.01 . 


\section{Results}

Out of 36,964 women with a valid HPV result, 87 (all HC2-positive) with a histologically confirmed diagnosis of high-grade squamous intraepithelial lesion or worse were excluded, leaving a total of 36,877 women for this analysis (mean age $=42.0$ years, 25 th percentile $=34$ years, 75 th percentile $=50$ years). Amongst 2833 HC2-positive women, 74.4\% (2108/2833) were confirmed to be positive for any of the 13 specific HPV infections using the PCR assay. Type-specific prevalences were comparable with those from a previous study in the Centre-South Italy that used the same design and HPV testing procedures. ${ }^{19,20}$

Table 1 shows the observed and expected numbers of women with single and multiple HPV infections ( 1 type, and $\geqslant 2$ types). Of the 2108 PCR-positive women, 430 (20.4\%) were infected with multiple types. The basic model included only age, study area and specific HPV type prevalence as covariates. The O/E ratio for infection with $\geqslant 2 \mathrm{HPV}$ types was 4.21 (95\% CI: 3.85-4.55). The Full model included, in addition to the covariates of the basic model, random intercepts for individual women, which represented unobserved host or environmental risk factors for HPV infection that are common to all types. The use of the Full model allowed the O/E ratio for infections with $\geqslant 2$ types to decrease to 1.21 (95\% CI: 1.13-1.30). The discrepancy between observed and expected counts was, therefore, not totally eliminated but greatly attenuated by the inclusion of individual random effects, although a small excess of infections with $\geqslant 2$ types was still present. As the number of women with three or more HPV infections was relatively small $(n=73)$, aggregated analyses only were presented for women with two or more HPV infections. In order to assess whether this clustering could be due to an excess of multiple infections involving certain combinations of HPV types, we investigated interactions between specific types.

Fig. 1 shows the observed versus expected occurrence for each two-way combination of 13 HPV types, under the Full model. No significant deviation of the observed from the expected counts was observed. None of the pairs of HPV types reached the chosen level of significance, $p$-value $<0.01$. However, a $p$-value $<0.05$, was observed for HPV31 with 58 ( $p$-value $=0.02$, O/E ratio $=0.32,99 \% \mathrm{CI}$ : 0.08-1.05) which were found together less often than expected. No evidence of a tendency to cluster for HPV types with genetic similarity was observed (data not shown).

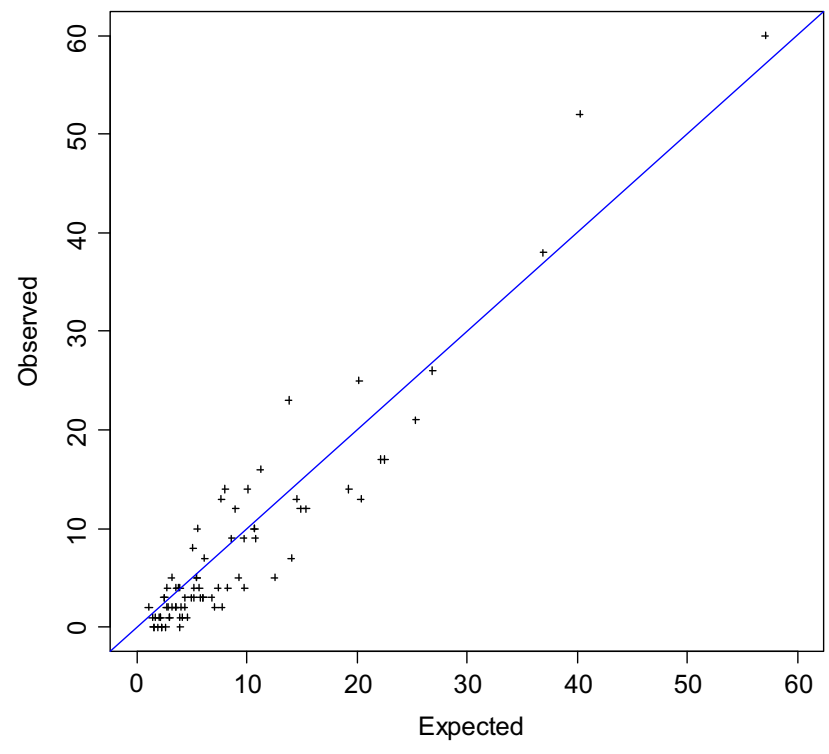

Fig. 1 - Observed versus Expected occurrence for 2-way combinations of $13 \mathrm{HPV}$ types. The New Technologies for Cervical Cancer screening study. Plus signs represent occurrences of HPV pairs. HPV pairs located in the upper triangle indicate positive clustering, while those located in the lower triangle represent negative clustering between the HPV types involved. None of the $p$-values for joint HPV infections was significant at the chosen significance level of 0.01 .

\section{Discussion}

The present analysis of the NTCC screening study confirmed that multiple HPV infections occurred more frequently than predicted by chance. ${ }^{1-7,9-12,21}$ The excess of multiple infections, however, was greatly reduced, though not completely eliminated, by controlling for sources of common correlation between HPV types. There was no evidence that specific HPV types have the tendency to be found more or less often than others in coinfections.

These observations are in agreement with previous findings that often reported a tendency of HPV types to cluster, as expected, ${ }^{22}$ but no excess or deficit of multiple infections involving specific HPV types. ${ }^{1-7,9-12,21}$ To note, our findings were substantially similar to those from studies carried out

Table 1 - Observed $(0)$ to Expected (E) ratio of multiple infections with the 13 HPV types, according to two models. The New Technologies for Cervical Cancer (NTCC) screening study.

\begin{tabular}{|c|c|c|c|c|c|}
\hline \multirow[t]{2}{*}{ No. of HPV types } & \multirow[t]{2}{*}{$\mathrm{O}$} & \multicolumn{2}{|c|}{ Basic model } & \multicolumn{2}{|c|}{ Full model } \\
\hline & & $E^{a}$ & $\mathrm{O} / \mathrm{E}(95 \% \mathrm{CI})^{\mathrm{a}}$ & $E^{b}$ & $\mathrm{O} / \mathrm{E}(95 \% \mathrm{CI})^{\mathrm{b}}$ \\
\hline 0 & 34,769 & 34363.0 & 1.01 (1.01-1.01) & 34751.0 & $1.00(1.00-1.00)$ \\
\hline 1 & 1678 & 2411.9 & $0.70(0.67-0.72)$ & 1771.4 & $0.95(0.91-0.99)$ \\
\hline$\geqslant 2$ & 430 & 102.2 & $4.21(3.85-4.55)$ & 354.6 & $1.21(1.13-1.30)$ \\
\hline
\end{tabular}


in different geographical areas, despite the large variation in the prevalence of HPV infection ${ }^{23}$ and of cervical disease. ${ }^{24,25}$

Statistical methods, based on mixed models or similar approaches, have been recently developed to control for the apparent positive association between all HPV types induced by common acquisition modalities. ${ }^{1,7,21,22}$ In the present study, we used subject-level random effects to account for sources of common correlation between all HPV types due to unobservable risk factors shared by all HPV infections. Random effects cannot distinguish between unobserved risk factors due to sexual behaviour of a woman or of her partner and other individual risk factors, such as immunological susceptibility. However, subject-random effects were particularly necessary in the present NTCC study to control for sources of common correlation between HPV types, as no information on sexual risk factors was collected for these women within the framework of the screening programmes.

Clusters involving certain combinations of specific HPV types have been previously reported, showing in some cases remarkably strong statistical associations. Such an apparent clustering was possibly caused by diagnostic artifacts of the HPV detection methods used. In particular, a previous analysis amongst women of the International Agency for Research on Cancer (IARC) HPV Prevalence Surveys (IHPS) that used GP5+/6+ PCR assays showed that cross-hybridisation between certain genetically related HPV types occurred when the Enzyme Immunoassay (EIA) method was used as genotyping procedure, this causing an apparent clustering of certain homologous HPV types in multiple infections. Conversely, the use of RLB nearly completely eliminated type clustering. ${ }^{1}$ RLB genotyping procedure was conceived to overcome suspected cross-hybridisation problems of the EIA procedure ${ }^{17}$ by using shorter probes (17-21 bases) and higher hybridisation temperature $\left(50^{\circ} \mathrm{C}\right)$, and it has now routinely replaced EIA. Although the results of the IARC study clearly confirmed the improved specificity of the RLB genotyping method, as compared to EIA, in assessing HPV types in multiple infections, the relatively small number of joint infections involving certain HPV types did not allow to completely rule out possible residual artifacts when using the RLB genotyping procedure.

The findings of the present analysis were based on the same HPV detection method, i.e. GP5+/6+ PCR followed by the RLB genotyping procedure, and on the same statistical approach of the IARC study. Compared to it, the NTCC study had a lower prevalence of overall $(5.7 \%$ out of 36,877 women versus $13.8 \%$ out of 6710 women) and of multiple (1.2\% versus $3.5 \%)$ HPV infections. In addition, only few women had multiple infections with $>2$ types $(N=73)$. However, the much larger absolute number of HPV-positive women (2108 of the NTCC versus 924 of the IARC study) and, consequently, of joint HPV infections, has allowed a more accurate analysis of the associations between combinations of HPV types, even those involving HPV types that are relatively less frequent in the general population. These results, together with those of the IARC surveys, confirm the improved specificity of the RLB, as compared to EIA, genotyping method and provide reassurance on lack of misclassification for HPV types in multiple infections as tested with GP5+/6+ PCR followed by the RLB procedure.
In summary, this analysis of the NTCC screening study showed minimal or no evidence of clustering between specific HPV types. The present findings, based on women aged 25-60 years attending population-based screening programmes in Italy, provide further evidence that the removal of certain HPV types through vaccination should not result in an indirect increase or decrease of the prevalence of other untargeted types.

\section{Role of the funding source}

This study was financially supported by the European Union (PREHDICT project FP7 grant agreement No. 242061) and by the Italian Ministry of Health (Progetto Speciale 'Valutazione di nuove tecnologie per lo screening del cervicocarcinoma follow-up' and L 138-2004 progetto 'NTCC - Nuove tecnologie per lo screening del cervicocarcinoma'). The funding sources did not have any role in the writing process of this manuscript.

\section{Conflict of interest statement}

None declared.

\section{Acknowledgement}

The work reported was partly undertaken while Dr. G. Ronco was hosted as Visiting Scientist by the International Agency for Research on Cancer.

\section{R E F E R E N C E S}

1. Vaccarella S, Franceschi S, Snijders PJ, et al. Concurrent infection with multiple human papillomavirus types: pooled analysis of the IARC HPV Prevalence Surveys. Cancer Epidemiol Biomarkers Prev 2010;19(2):503-10.

2. Thomas KK, Hughes JP, Kuypers JM, et al. Concurrent and sequential acquisition of different genital human papillomavirus types. J Infect Dis 2000;182(4):1097-102.

3. Liaw KL, Hildesheim A, Burk RD, et al. A prospective study of human papillomavirus (HPV) type 16 DNA detection by polymerase chain reaction and its association with acquisition and persistence of other HPV types. J Infect Dis 2001;183(1):8-15.

4. Rousseau MC, Pereira JS, Prado JC, et al. Cervical coinfection with human papillomavirus (HPV) types as a predictor of acquisition and persistence of HPV infection. J Infect Dis 2001;184(12):1508-17.

5. Chaturvedi AK, Myers L, Hammons AF, et al. Prevalence and clustering patterns of human papillomavirus genotypes in multiple infections. Cancer Epidemiol Biomarkers Prev 2005;14(10):2439-45.

6. Mendez F, Muňoz N, Posso H, et al. Cervical coinfection with human papillomavirus (HPV) types and possible implications for the prevention of cervical cancer by HPV vaccines. J Infect Dis 2005;192(7):1158-65.

7. Chaturvedi A, Katki H, Hildesheim A, et al. Human Papillomavirus infection with multiple types: pattern of coinfection and risk of cervical disease. J Infect Dis 2011;203(7):910-20. 
8. Vaccarella S, Franceschi S, Herrero R, et al. Sexual behavior, condom use and HPV: pooled analysis of the International Agency for Research on Cancer HPV Prevalence Surveys. Cancer Epidemiol Biomarkers Prev 2006;15(2):326-33.

9. Franco EL, Villa LL, Sobrinho JP, et al. Epidemiology of acquisition and clearance of cervical human papillomavirus infection in women from a high-risk area for cervical cancer. J Infect Dis 1999;180(5):1415-23.

10. Molano M, van den Brule AJ, Plummer M, et al. Determinants of clearance of human papillomavirus infections in Colombian women with normal cytology: a population-based, 5-year follow-up study. Am J Epidemiol 2003;158(5):486-94.

11. Vaccarella S, Franceschi S, Herrero R, et al. Clustering of multiple human papillomavirus infections in women from a population-based study in Guanacaste, Costa Rica. J Infect Dis 2011;39:1179-89.

12. Vaccarella S, Plummer M, Franceschi S, et al. Clustering of human papillomavirus (HPV) types in the male genital tract: the HPV in men (HIM) study. J Infect Dis 2011;204(10):1500-4.

13. Ronco G, Giorgi-Rossi P, Carozzi F, et al. Results at recruitment from a randomized controlled trial comparing human papillomavirus testing alone with conventional cytology as the primary cervical cancer screening test. J Natl Cancer Inst 2008;100(7):492-501.

14. Ronco G, Segnan N, Giorgi-Rossi P, et al. Human papillomavirus testing and liquid-based cytology: results at recruitment from the new technologies for cervical cancer randomized controlled trial. J Natl Cancer Inst 2006;98(11):765-74.

15. Ronco G, Giorgi-Rossi P, Carozzi F, et al. Human papillomavirus testing and liquid-based cytology in primary screening of women younger than 35 years: results at recruitment for a randomised controlled trial. Lancet Oncol 2006;7(7):547-55.
16. Jacobs MV, Roda Husman AM, van den Brule AJ, et al. Groupspecific differentiation between high- and low-risk human papillomavirus genotypes by general primer-mediated PCR and two cocktails of oligonucleotide probes. J Clin Microbiol 1995;33(4):901-5.

17. van den Brule AJ, Pol R, Fransen-Daalmeijer N, et al. GP5+/6+ PCR followed by reverse line blot analysis enables rapid and high-throughput identification of human papillomavirus genotypes. J Clin Microbiol 2002;40(3):79-787.

18. Gelman A, Carlin JB, Stern HS, Rubin DB. Bayesian Data Analysis. 2nd ed. Boca Raton, Florida: Chapman \& Hall/CRC publisher; 2004.

19. Giorgi-Rossi P, Bisanzi S, Paganini I, et al. Prevalence of HPV high and low risk types in cervical samples from the Italian general population: a population based study. BMC Infect Dis 2010;10:214.

20. Giorgi-Rossi P, Chini F, Bisanzi S, et al. Distribution of high and low risk HPV types by cytological status: a population based study from Italy. Infect Agent Cancer 2011;6(1):2.

21. Plummer M, Schiffman M, Castle PE, Maucort-Boulch D, Wheeler CM. A 2-year prospective study of human papillomavirus persistence among women with a cytological diagnosis of atypical squamous cells of undetermined significance or low-grade squamous intraepithelial lesion. $J$ Infect Dis 2007;195(11):1582-9.

22. Plummer M, Vaccarella S, Franceschi S. Multiple human papillomavirus infections: the exception or the rule? J Infect Dis 2011;203(7):891-3.

23. De Vuyst H, Clifford G, Li N, Franceschi S. HPV infection in Europe. Eur J Cancer 2009;45(15):2632-9.

24. Ronco G, van Ballegooijen M, Becker N, et al. Process performance of cervical screening programmes in Europe. Eur J Cancer 2009;45(15):2659-70.

25. Ronco G, Antilla A. Cervical cancer screening in Europe changes over the last 9 years. Eur J Cancer 2009;45:2629-31. 